Cahiers de recherches médiévales

\title{
Emmanuèle Baumgartner (1940-2005)
}

Jean Dufournet

\section{(2) OpenEdition}

Journals

Édition électronique

URL : https://journals.openedition.org/crm/841

DOI : $10.4000 / \mathrm{crm} .841$

ISSN : 1955-2424

Éditeur

Honoré Champion

Édition imprimée

Date de publication : 30 mars 2006

Pagination : i-vi

ISSN : 1272-9752

\section{Référence électronique}

Jean Dufournet, «Emmanuèle Baumgartner (1940-2005) 》, Cahiers de recherches médiévales [En ligne], 13 spécial | 2006, mis en ligne le 03 avril 2009, consulté le 15 décembre 2022. URL : http:// journals.openedition.org/crm/841; DOI : https://doi.org/10.4000/crm.841 


\section{酷M}

\section{INTRODUCTION}

Ce numéro spécial des Cahiers de Recherches Médiévales recueille les contributions de journées d'études qui se sont tenues à Paris, dans les locaux de l'université Denis Diderot, les 2 et 3 décembre 2005. Cette rencontre s'inscrit dans un programme de recherches «Entre Moyen Âge et Renaissance » animé en collaboration par deux équipes : Tradition Antique et Modernité de Paris-VII-Denis Diderot et Littérature et Histoire de l'université d'Orléans. Les premiers travaux, portant sur l'Héroïque, avaient déjà fait l'objet d'un numéro spécial des $C R M$, en 2004, à la suite d'une table ronde ayant eu lieu à Orléans, organisée en collaboration avec Denis Bjaï. C'est donc bien dans une continuité que la publication de ces numéros spéciaux est à considérer.

Le but visé est de participer à des recherches qui, si elles émergent peu à peu depuis quelques temps, méritent d'être largement développées. On s'accorde en effet de plus en plus à considérer le découpage académique Moyen Âge/Renaissance comme artificiel. Mais il n'est pas non plus complètement vide de sens. Il est donc nécessaire que les spécialistes de chacune de ces périodes se rencontrent, afin de travailler en commun et d'échanger leurs données, leurs analyses et leurs hypothèses, en réfléchissant aussi de concert pour déterminer le plus précisément possible ce qui réunit et ce qui différencie les périodes médiévale et humaniste d'un point de vue culturel et sur le terrain de l'histoire des idées et des mentalités. Pour ce faire, il existe beaucoup de portes d'entrée : nous nous sommes d'abord orientés vers des voies littéraires, en nous penchant sur des textes ressortissant en priorité au domaine des lettres. Mais ceci n'a rien d'une position de principe et, dans l'avenir, des sujets plus interdisciplinaires seront abordés.

Il nous a semblé qu'une figure historique venue de l'Antiquité présentait un intérêt particulier dans l'étude des articulations entre d'une part un temps qui plonge largement ses racines dans une Antiquité latine qu'il vénère, et d'autre part une époque qui prétend 'redécouvrir' les lettres et la culture antiques et qui, effectivement, met en lumière une partie de cette culture, et, de façon générale, tente de relire cette Antiquité avec de nouveaux outils, certains effectivement neufs, d'autres directement hérités de la fin du Moyen Âge.

Le 'César médiéval' n'a pas jusqu'à présent suscité un profond intérêt dans la critique. Si l'on exclut l'ouvrage de J. Beer, A medieval Caesar, publié en 1976 et celui de J. Leeker, Die Darstellung Cäsars in den romanischen Literaturen des Mittelalters, paru en 1986, peu d'articles et de monographies sont consacrés à ce personnage. Il est vrai que les figures emblématiques d'Alexandre, d'Arthur et de Charlemagne occupent le premier rang dans la représentation du pouvoir au Moyen Âge et qu'en outre, elles sont toutes trois inscrites dans une solide tradition littéraire: respectivement, les divers Romans d'Alexandre, les romans dits arthuriens, la chanson de geste. César en revanche connaît une fortune littéraire bien plus modeste durant le Moyen Âge : à titre significatif, seul un texte complet dont il est le héros nous est parvenu : le Roman de Jules César composé vers le milieu du 
XIII ${ }^{\mathrm{e}}$ siècle par Jean de Thuin et dont il existe une version en prose, probablement du même auteur, l'Histoire de Jules César, dérimage du Roman. Le Roman de Jules César se présente comme une adaptation de La Pharsale de Lucain, texte bien connu des médiévaux (plus de deux cents manuscrits), une des sources des Faits des Romains qui, avec l'Histoire ancienne jusqu'à César, est un des textes principaux en matière d'histoire romaine médiévale. Si l'on considère ce texte dans la perspective des 'romans antiques', dont les premiers se présentent très explicitement comme des traductions d'épopées latines, une question se pose : pourquoi, alors que l'épopée de Lucain était très connue et recopiée, s'est-il écoulé un temps aussi long entre le Roman de Thèbes, l'Eneas et le Roman de Jules César?. Silvère Menegaldo se penche sur cette question qui, de fait, en pose une autre : celle du statut de Jules César en tant que personnage de roman ou d'épopée. Michelle Szkilnik reprend à son tour cette problématique, mais dans une perspective différente, en comparant deux romans tardifs, quasiment contemporains, qui ont en commun d'être aux confins de l'histoire et du roman : Le Jouvencel de Jean de Bueil, rédigé entre 1461 et 1468 et le Roman de Perceforest. En outre, l'un des manuscrits du Perceforest et l'un des manuscrits du Jouvencel ont été copiés pour le même mécène, Louis de Bruges, seigneur de la Gruthuyse, ce qui ajoute un argument de justification pour comparer les deux textes. La conclusion de M. Szkilnik rejoint en partie celle de S. Menegaldo sur ce que l'on pourrait appeler un 'échec' d'un César personnage littéraire : le Perceforest, par exemple, ne parvient à présenter César qu'en grand conquérant, au demeurant plutôt négatif, parce que perdu par son orgueil et son ambition. César demeure donc un personnage exemplaire, positif, négatif ou ambigu, qui n'arrive pas à s'inscrire pleinement, en tant qu'actant, dans un texte de fiction, parce que, peut-être, il est un personnage «trop historique».

Est-ce le même type d' 'échec' qui fait de César un personnage très peu présent dans le Violier des histoires romaines, traduction tardive des Gesta romanorum? Sans doute pas, car, comme le montre Denis Hüe, les enjeux de ce recueil d'exempla sont bien différents et l'on aboutit à une figure ambiguë, voire contradictoire : abstraitement représenté, César est un modèle, gardien du droit et de la justice. Mais dès qu'il est question de lui en tant qu'être humain, c'est l'orgueil qui domine et l'auteur du Violier montre un César impie, toujours sourd et aveugle aux injonctions et aux signes du destin, toujours coupable de démesure. C'est, comme le souligne D. Huë, une sorte de fatalité qui caractérise son cheminement, fatalité qui le montre à la fois exemplaire et bien proche de chacun de nous, comme le souligne le commentaire qui parle du nombre des baptisés incapables de tenir leurs engagements.

Pour rester à la fin du Moyen Âge, dans le cadre d'une écriture didactique telle celle de Christine de Pizan, l'on peut faire un constat analogue : César demeure un personnage fondamentalement exemplaire, mais sous divers aspects : conquérant, grand chef militaire admiré de ses soldats, homme bon et vertueux, savant capable de prouesses intellectuelles et de projets d'envergure dans le domaine culturel et scientifique. Même si l'orgueil du personnage est déploré, il demeure positif et son classement, après Jacques de Longuyon, dans la liste des neuf preux est un topos souvent repris. Chez Christine, l'emploi d'un personnage historique, puisé dans des sources autorisées, est en rapport avec le portrait du prince idéal, tel qu'elle l'envisage dans Le Livre de Prudence. Dans ce contexte, on peut penser que Jules 
César ne présente rien de très particulier en regard d'autres preux, surtout Alexandre, lui aussi conquérant, puissant, sage et savant. Cependant, César a une spécificité par rapport au Macédonien : il appartient à la lignée troyenne et se trouve être à ce titre un des personnages les plus importants de l'histoire romaine par l'attachement que Christine, comme d'autres auteurs de la fin du Moyen Âge, porte à la légende des origines troyennes de la royauté française.

La figure exemplaire de César telle qu'elle apparaît régulièrement dans la littérature française a, de fait, été préparée avant le XIII ${ }^{\mathrm{e}}$ siècle et les Faits des Romains, dans une certaine littérature historique médiolatine et sa traduction : celle qui met en place la légende d'Arthur. C'est ce que montre bien la contribution de Catherine Croizy-Naquet, qui se penche en particulier sur le Roman de Brut de Wace. Ce dernier fournit les traits les plus marquants de César qui, fragmentés, diffractés, fonctionnent le plus souvent comme éléments de comparaison renvoyant à un type de pouvoir ou à un type de savoir et de comportement, et cela Wace l'exprime en langue romane. De figure mythique, César devient donc le support d'une figure stylistique, la comparaison, ou l'illustration d'une qualité, ce qu'il va largement rester pour tout le Moyen Âge. La contrepartie est que, dès le Brut, César est, comme le montre fort bien C. Croizy-Naquet, véritablement 'chassé' de l'espace romanesque, occupé par Arthur. Position lourde de conséquence pour l'avenir littéraire du personnage.

Certains auteurs, utilisant Jules César comme personnage exemplaire, insistent sur ses qualités intellectuelles. Mais il s'agit souvent de prouesses (dicter plusieurs lettres à la fois) ou de projets importants (bibliothèque, renouvellement du droit), ou bien encore de valeurs scientifiques (le calendrier et le bissexte). Curieusement, alors que l'œuvre de César est connue, utilisée par certains auteurs comme celui des Faits des Romains, la figure de l'écrivain demeure somme toute assez diaphane, sauf peut-être chez Pétrarque, qui estime l'historien, mais ne le range quand même pas en tête de ses auteurs préférés. À la fin du Moyen Âge cependant, les Commentaires font l'objet d'un manifeste regain d'intérêt, dont témoignent diverses traductions. C'est à l'une des plus importante, celle de Robert Gaguin, que Frédéric Duval s'intéresse ici, en se penchant donc sur la réception du De bello Gallico. La cour de Bourgogne va jouer un rôle important dans cette diffusion de la Guerre des Gaules en français. Une traduction des Commentaires, dans laquelle les Belges, ancêtres des Bourguignons, sont exaltés, est réalisée en 1473 par Jean Duchesne à l'attention de Charles le Téméraire. En 1485, Robert Gaguin, qui connaissait la traduction de Duchesne, offrit au jeune roi Charles VIII une nouvelle version française appelée à une diffusion considérable et durable, grâce en particulier au relais de l'imprimerie. Comment expliquer la pérennité de ce texte à une époque où l'appréhension de l'Antiquité romaine se modifie, tout comme la place des Gaulois dans l'historiographie nationale? C'est en étudiant les realia romana et plus largement des termes de civilisation romaine inscrits dans le texte que F. Duval se propose de répondre à cette question. Il apparaît que Gaguin s'inscrit dans un mouvement de réaction contre les traductions savantes et leurs latinismes, devenus incompréhensibles pour beaucoup : il y préfère cultiver un français de registre aussi élevé que l'était le latin de César. Mais cette norme que Gaguin se proposait d'appliquer au français dans le cadre d'une traduction ne put s'imposer face aux tenants d'un enrichissement de la langue vernaculaire par emprunt au latin. 
Pour la Renaissance, l'histoire romaine fournit au souverain des modèles et des contre-modèles. Sandra Provini observe que Charles VIII suscite des comparaisons avec César, afin de suggérer une translatio imperii de l'empire romain à la royauté française ; au contraire, comme Louis XII entend apparaître, en Italie notamment, comme un libérateur de la tyrannie, les poètes composant en son honneur évitent de telles comparaisons. François $\mathrm{I}^{\mathrm{er}}$, à son tour, favorise les parallèles avec César. Dans la seconde partie du XVI ${ }^{\mathrm{e}}$ siècle, on relit l'histoire antique pour comprendre l'actualité. Comme le montre Emmanuel Buron, Jodelle établit une analogie entre la situation où se trouvait Charles IX avant le conflit religieux et celle de César devant le Rubicon, pour inciter le jeune roi à l'analyse critique de la conjoncture et à la réflexion sur la bonne conduite politique. Denis Bjaï remarque qu'Étienne Pasquier médite sur les troubles civils du XVI ${ }^{\mathrm{e}}$ siècle à partir du De bello ciuili, et Emmanuel Buron qu'à la fin du siècle, le rapprochement entre Henri IV et César devient un lieu commun.

En effet, la Renaissance, éprise de gloire, a la passion des grands hommes, mais, à l'exemple de Plutarque, elle guette leurs faiblesses. Côté lumière, César réunit des qualités qui font de lui à la fois un homme de guerre, un homme d'Etat et un homme de lettres. Il est d'abord «le vray et souverain patron de l'art militaire »selon l'expression de Montaigne - et le parangon des hommes d'actions, sobre, vif, courageux, prudent; il est ensuite celui qui a mis fin aux guerres civiles et qui a pourvu une société exsangue d'un pouvoir stable, ce qui le rapprocherait de Henri IV; il a enfin communiqué ses réflexions dans des Commentaires, témoignant du passé lointain de la France et fournissant à la postérité le «breviaire de tout homme de guerre ». Il apparaît donc comme l'homme total, «Vaillant et scavant tout ensemble, / Qui le jour domtoit ses haineux, / Et la nuit escrivoit sa gloire », comme dit Ronsard. Côté ombre, César est un «brigand»- pour reprendre le mot de Marcellus cité par Montaigne -, un ambitieux, prêt à écarter tous les obstacles et à enfreindre le droit si nécessaire. Denis Bjaï et Daniel Ménager commentent ici le rappel qui est fait, au XVI ${ }^{\mathrm{e}}$ siècle, les vers de Sophocle où César trouvait une justification. Il a usé de la corruption et s'est emparé des deniers publics. Comme le souligne Françoise Charpentier, Montaigne reproche à César d' "avoir voulu chercher sa gloire de la ruine de son pays ». L'appétit de pouvoir conduit à instaurer un régime autoritaire. Parce qu'Étienne de La Boétie conçoit la république comme un idéal, il récuse la façon dont les humanistes voient César et il en fait l'archétype du tyran. Le suicide de Caton est une protestation en faveur de la liberté agonisante. Françoise Charpentier montre que, chez La Boétie, César est coupable d'un autre travers: sa « venimeuse douceur» confine à l'hypocrisie.

La Renaissance insiste donc sur l'ambiguïté de César - pour reprendre un terme judicieusement employé par Bénédicte Boudou : c'est un héros mais aussi un tyran, un soldat courageux mais aussi un politicien rusé. Son cas nous invite à méditer sur l'ambivalence des comportements: quelles limites tracer entre habileté et rouerie ? entre douceur et hypocrisie ? entre ambition et magnanimité ?

La pesée d'une âme, dans le cas de César, est un peu mesquine : Pétrarque dirait qu'elle trahit l'envie. L'imperator, en effet, rend vain le décompte des vertus et des vices. Comme il n'avait souci de ressembler qu'à lui-même, on imagine qu'il faisait peu de cas des jugements moraux. Il évolue à des hauteurs où les notions de 
bien et de mal perdent leur pertinence. Selon Françoise Charpentier, Montaigne reconnaîtrait la "personnalité géniale» de César, et, d'après Daniel Ménager, l'évocation par Pline de sa légèreté voltigeante signale «la présence du génie ». César est une figure de la démesure. Comme le rappelle Daniel Ménager, l'épilepsie fait soupçonner son commerce avec le Ciel. Si César suscite sans cesse la rêverie des hommes, c'est qu'il jouxte le divin.

En dessinant les contours qui, du $\mathrm{XII}^{\mathrm{e}}$ au $\mathrm{XVI}^{\mathrm{e}}$ siècle, ont été donnés à la personnalité complexe de César, les études réunies dans ce volume contribuent à une histoire des représentations et confirment que le Moyen Âge et la Renaissance se rencontrent dans une commune fascination pour l'Antiquité.

Bruno Méniel - Bernard Ribémont 